\title{
Including coronary ostia in patient-specific 3D models of the whole aortic valve apparatus, derived from TEE, for biomechanical simulations.
}

\author{
M Loureiro-Ga',2*, C Veiga1, G Fdez-Manin², F Calvo-Iglesias³, \\ V Alfonso Jimenez ${ }^{3}$, P Pazos $^{3}$, A Iñiguez ${ }^{3}$ \\ 1. Department of Cardiology. Galicia Sur Health Research \\ Institute (IIS Galicia Sur). SERGAS-UVigo. Hospital \\ Alvaro Cunqueiro, 36212 Vigo, Spain \\ 2. Department of Applied Mathematics II. University of Vigo. \\ Higher Technical School of Telecommunications \\ Engineering, 36210 Vigo, Spain \\ 3. Department of Cardiology. Complexo Hospitalario \\ Universitario de Vigo (CHUVI). Hospital Alvaro \\ Cunqueiro - SERGAS, 36212 Vigo, Spain
}

\begin{abstract}
There is an increasing interest in the numerical modeling and simulation of the aortic valve behavior and functioning, on the different stages involved as healthy, stenotic or replacement procedure. As echocardiography is a ubiquitous and economic modality, the geometric model construction based on such images is therefore of major interest. In this paper, a new patient-specific approach for modeling the complete aortic valve apparatus - derived from parameters extracted from 3D transesophageal echocardiographs -that includes for the first time the left ventricle outflow tract and the coronary ostia, both crucial for proper assessment of valve biomechanical behavior, is presented. An innovative method for characterizing coronary pressures from patient-specific clinical data, to be used as boundary conditions for the numerical simulation is also described. Results from experiments were presented to evaluate the novel aspects of the model, that permits to compare the existing models (non-coronary model NCM) and the proposed new coronary model (CM). Variations of displacement and stress on each leaflet prove the need of considering leaflet asymmetry. Computed quantities in the results sections are within the range of physiological data. This permits to conclude that the proposed model of the aortic valve apparatus improves on previous ones by considering this extremely complex structure in greater detail.
\end{abstract}

$\overline{{ }^{*} \text { Corresponding Author: maloureiro@uvigo.gal }}$ 


\section{INTRODUCTION}

The aortic valve (AV) is a structure located between the left ventricle and the aorta that connects the left ventricular outflow tract (LVOT) with the ascending aorta. The main parts of the AV are the valve leaflets and the aortic sinuses (sinuses of Valsalva). The AV opens and closes to ensure that blood flows in only one direction in systolic ejection [5] when function is healthy. For the purposes of this research, we denote the AV and related parts including the LVOT and the coronary ostia — as the AV apparatus, depicted in Figure 1a.

AV diseases are a major cause of morbidity and mortality in the elderly in western countries [28]. Given its importance, since the first sketches by Leonardo da Vinci (16th century) [37], the AV has attracted great and growing interest within several scientific disciplines, as proper functional assessment of the AV is crucial. Even though the new imaging modes provide many sights to the AV, its complete and comprehensive assessment remains a challenge [19].

The understanding of the optimum function of the healthy aortic valve is essential in interpreting the effect of pathologies in the region, and in devising effective treatments to restore the physiological functions [43]. In this scenario, each new insight to the AV are of crucial importance. Using numerical simulation to assess the AV function can potentially throw new light on AV behavior. Although several numerical simulation studies have been conducted for academic purposes using very simplified geometries $[8,14,16]$, realistic numerical simulation requires a very detailed geometry.

In recent decades, numerical simulation has been increasingly used to support clinical practice [42]. Regarding the heart valves, several studies have been published with interest topics such as analyzing patient-specific transcatheter AV implantations [4, 30, 38, 39], analyzing the motion of a parametrical model of the mitral valve [46], modelling bicuspid and stenotic AVs [12,26,31,48] or assessing coronary flow improvements after TAVI [27]. Obtaining an accurate geometric model of the patient-specific AV apparatus, however, is required to carry on a numerical simulation that could support clinical decision making. Geometric model construction based on information extracted from images, such as those shown in Figure 1b, is therefore a crucial part of the process.

Significant progress has been made in the AV modeling field. The first leaflet geometric models were published at the end of the 1970s [47]. Later, Thubrikar [44] studied the design of the AV to ensure appropriate sealing of the leaflets; since then, many other papers have made further contributions to the literature on this topic [13,20,22,33,34], which is attracting growing interest. Proposals include a geometric model for functional AV leaflets developed from 2D TTE considering certain geometric assumptions [20] and a 3D parametric morphological representation of the aortic sinus [33]. Another development was 3D TEE modeling of the AV that included the leaflets and sinuses [13] and the ascending aorta considering curvature [6], which affects blood dynamics downstream from the AV [18]. A recent publication explains how 3D TEE as a function of time (3D+t TEE) obtains AV dimensions (leaflets and sinuses) and describes how to build a scalable finite element (FE) model [22]. 
Nonetheless, those models are based on an incomplete AV apparatus description. Recent publications consider more complex geometries: a geometric AV model that includes the coronary arteries, derived from a combination of CT and MRI is described in [30]; simulations in reconstructions of patient-specific cases in which it is possible to distinguish between leaflet tissue and calcified plaques is done in [2]. Despite the evolution of cardiac imaging methods in recent decades, echocardiography continues to be the most frequently performed imaging study in cardiology, given its capacity to yield a detailed and accurate evaluation of AV structure and function. Deriving new clinical usages and applications of this longstanding method is therefore of major interest.

Regarding our patient-specific geometric model based on echocardiographic images, below we describe a semiautomated process for obtaining the parameters and building the geometry of a new model that considers leaflet asymmetry, interleaflet triangles and the aortic sinus together with thickness parameters; it also takes into account the curvature of the ascending aorta, the LVOT, the coronary ostia and a small portion of the coronary artery. The inclusion of these parameters is justified on the basis that they may significantly affect biomechanical behavior and fluid dynamics of the aortic root [36].

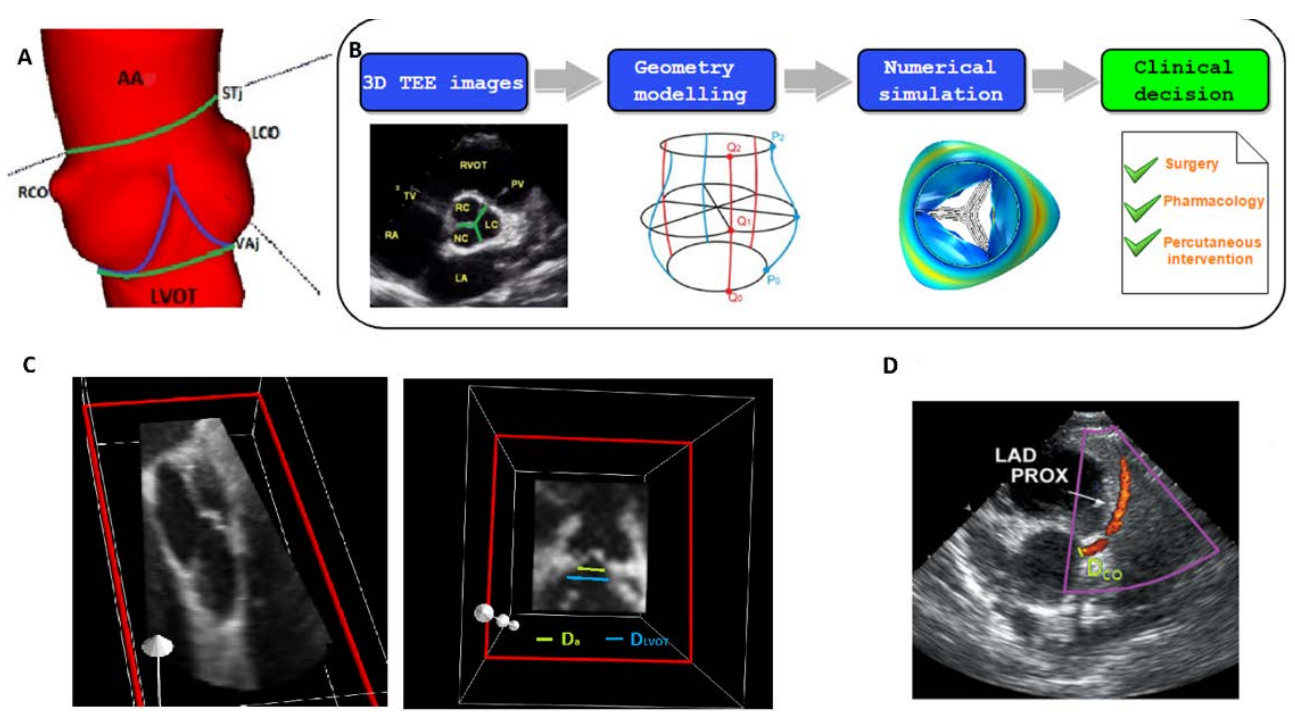

Figure 1: A) the AV apparatus in 3D. B) the methodological pipeline of geometric modelling and numerical simulation of patient medical images of the AV apparatus leads to improved clinical decision making due to a better understanding of AV diseases. C) 3D views of the AV from a 3D TEE video sequence. Images were acquired with a 3D TEE transducer and exported in cartesian coordinates. A macro was written to open, display and measure the images. D) Image showing where the proximal left anterior descending (LAD PROX) using the echo-Doppler option to assess coronary artery diameter Dco. 


\section{MATERIALS AND METHODS}

\subsection{Imaging the AV apparatus}

Building the AV apparatus geometry requires patient-specific measurements. Some authors have used 2D TTE [20,33], but recent advances enable geometry to be captured as a function of time using multiplane TEE data [15]; these 3D+t TEE images can significantly improve the geometry of the model. Using multiplane TEE combined with echo-Doppler improves visualization of the coronary arteries, which means that echo-derived geometric models can be enriched by including the coronary ostia and a portion of each artery (see Figure 1c for several transesophageal views of multiple planes of the AV).

Patient-specific data were obtained at Alvaro Cunqueiro Hospital (Vigo, Spain). Several studies were performed of the patients using the iE33 system (Philips Medical Systems, Andover, MA) equipped with a fully sampled matrix-array TEE transducer under a protocol approved by the local Institutional Review Board. Images of the AV, the proximal ascending aorta and the proximal left anterior descending coronary artery were obtained over two consecutive cardiac cycles.

\subsubsection{Measuring the AV apparatus}

$\mathrm{AV}$ measurements from the patient images captured in late diastole were obtained in accordance with the stages published elsewhere [22]. A macro script in ImageJ [41] was used to process the images and obtain 21 anatomical landmarks [22]. The script opens the images in different views and allows the selection of landmarks which are measured to obtain their size as shown in Figure 1C. Parameters described in the state of art include: HR, HR, HN FR, FL, FN to reconstruct the leaflets; Da, Dstj, Daa, Hsin, Hv, Haa, a, b, c, d, e, f, $\alpha, \beta, \gamma$ to construct the aortic sinus and interleaflet triangles. Further details about the interpretation of those parameters can be found in Figure 2 and in publications such as [7,20,21,22,40].

In addition to those known landmarks, we extracted further information for the purpose of this research, namely, the LVOT diameter and the diameter for the coronary ostia, using the clinical procedure [3].

- $\quad$ LVOT diameter $-\mathrm{D}_{\mathrm{LVOT}}$ : can be measured at a fixed point at a distance (height) from the base of the AV (the ventriculoaortic junction; $\mathrm{VAj}$ ). This height parameter is noted as $\mathrm{H}_{\text {LVOT. }}$. $\mathrm{D}_{\text {LVOT }}$ measurement is depicted in blue in Figure 1C. In that case, the height distance $\mathrm{H}_{\mathrm{LVOT}}$ from $\mathrm{D}_{\mathrm{LVOT}}$ to Da was $5 \mathrm{~mm}$.

- Coronary ostia diameter (Dco): can be measured using the echo-Doppler function to image flow in the coronary artery as shown in Figure 1D.

The values for those new measurements can be found in Table 1 . The use of all those measurements to build a patient-specific 3D geometry model is detailed in the next section. 
a

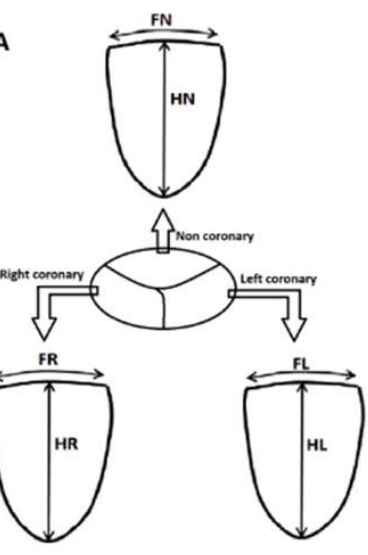

B

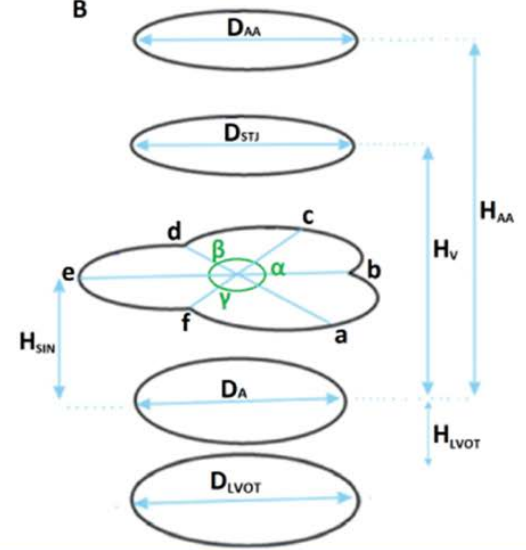

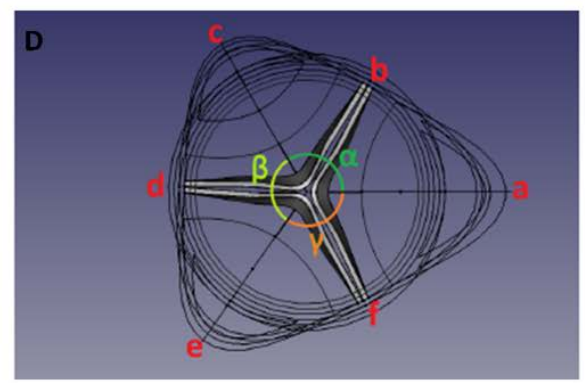
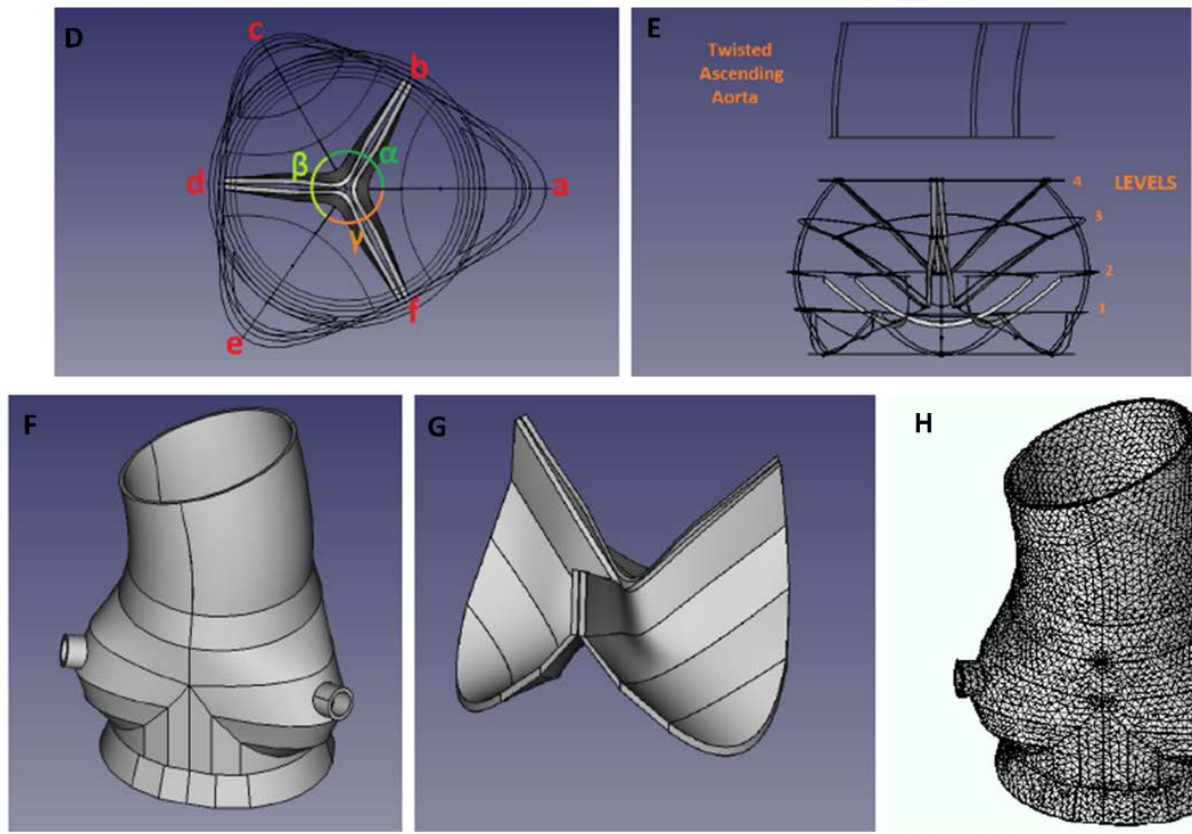

H

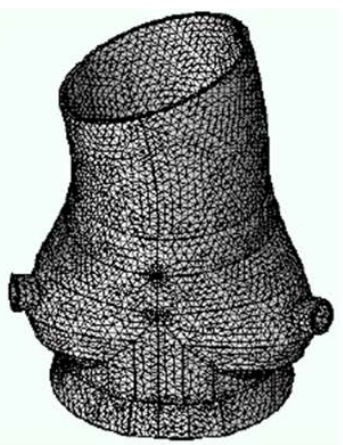

Figure 2: A) The 6 landmarks used for leaflet reconstruction: the three heights of the right, left and noncoronary leaflets ( $H R, H R$ and $H N$, respectively) and the three free-edge lengths of the right, left and noncoronary leaflets (FR, FL and FN, respectively). B) A sketch of the geometry shown the 15 parameters (Da, Dstj, Daa, Hsin, Hv, Haa, a, b, c, d, e, f, $\alpha, \beta, \gamma)$ and the new DLVOT and HLVOT parameters. D) Top view of the sketch used to reconstruct the geometry. E) basic model as proposed in Morganti et al. [13] with the ascending aorta improved as in Haj-Ali et al. [11]. F) final AV apparatus reconstruction that includes leaflets, aortic sinus, interleaflet triangles, ascending aorta, LVOT, coronary ostia and a small portion of the coronary arteries. G) interior view of final AV apparatus reconstruction. $H$ ) a finite element mesh of the AV apparatus geometry. 


\subsection{Patient-specific geometric model}

The reconstruction of the AV leaflets, using the six length measurements mentioned in the previous section, was based on an existing leaflets model [20]. The main difference of our work with that study is that we consider the leaflets asymmetry; symmetric leaflets would prevent the model from capturing the varying biomechanical behavior of the leaflets [7]. The three leaflets were thus assumed to have different sizes and to lie at different angles from each other $(\alpha, \beta, \gamma)$ in the circumferential direction of the AV, as shown in Figure 2D.

To obtain the basic geometry of the aortic sinus and the interleaflet triangles, an existing 3D parametric model [33] was implemented. Since the interleaflet triangles have to be reconstructed from the bottom part of the aortic sinus to ensure proper matching of the solids, the surface was split into different parts to reconstruct both leaflets and sinus (this explains why the interleaflet triangles are composed of several small parts). The model was improved by modeling a portion of the ascending aorta [7], as can be observed on the right of Figure 2F.

\subsubsection{New model of the complete AV apparatus}

We introduced two main improvements to the existing basic geometric model by including coronary and LVOT components.

The first improvement was to include the coronary ostia together with a small portion of the coronary arteries. The left coronary ostium was created at positions a in Figure 2D and height Hsin in Figure 2B, i.e. the positions of maximal expansion of the aortic sinuses. A portion of the coronary artery was modeled as a hollow cylinder, perpendicular to the surface in that position, with an interior diameter Dco (measured as can be seen in Figure 2C), a thickness thC and a length Lca. Since the right coronary is less accessible echocardiographically, it was assumed to lie at the same height; the same procedure was used to model the right coronary, rotating the cylinder with the corresponding angle $(-\gamma)$ to point $\mathrm{e}$ in Figure 2D.

The second improvement was the inclusion of the LVOT, for which we needed to know the angle with the vertical at the level of the VAj:

$$
\psi=\arctan \left(\frac{D_{L V O T}-D_{a}}{2 H_{L V O T}}\right)
$$

The FreeCAD free software package was used to build the geometry [9]. A script to automate the model building procedure was written and incorporated in FreeCAD. Considered in our implementation were general tissue thicknesses for the leaflets (thL) and the aortic sinus (thV) of $0.5 \mathrm{~mm}$ and $1 \mathrm{~mm}$, respectively. Table 1 provides summary details of the additional parameters required to improve the AV apparatus geometry. 
Table 1. Patient-specific parameters values. First part: list of the 7 new additional parameters included in the proposed model. Second part: values and times, in seconds, obtained from the instantaneous wave-free ratio study for a cardiac cycle of $0.85 \mathrm{~s}$.

\section{GEOMETRIC PARAMETERS}

\begin{tabular}{llc}
\hline Abbreviation & Description & \multicolumn{1}{c}{ Value (mm) } \\
\hline DLVOT & Diameter of the LVOT & 27 \\
Dco & Diameter of the left-right coronary artery & 3 \\
HLVOT & $\begin{array}{l}\text { Distance (height) under the VAj where the DLVOT is } \\
\text { measured }\end{array}$ & 5 \\
Lca & Length of the coronary arteries & 2.5 \\
thL & Leaflet thickness & 0.5 \\
thV & $\begin{array}{l}\text { Thickness of the aortic sinus, ascending aorta and LVOT } \\
\text { tissue }\end{array}$ & 1 \\
thC & Thickness of the coronary artery tissue & 0.5 \\
\hline
\end{tabular}

PRESSURE PARAMETERS

$\mathrm{t}(\mathrm{s})$

\begin{tabular}{lllllll} 
& \multicolumn{9}{c}{ 1st cardiac cycle } & \multicolumn{1}{c}{ 2nd cycle } \\
\hline $\mathrm{tP}$ & $\mathrm{tR}$ & - & $\mathrm{tT}$ & AV closure & - & $\mathrm{t}_{\mathrm{P}}$ \\
\hline 0 & 0.2 & 0.3 & 0.45 & 0.55 & 0.65 & 0.85 \\
62 & 90 & 100 & 120 & 100 & 75 & 62
\end{tabular}

\subsection{Biomechanical simulation}

In our study, a FE simulation was configured intermediately between structural and fullycoupled FSI solving a two cardiac cycles time-dependent problem for blood flow and a parametric study for cardiac tissue, which was loaded with the tensions of the fluid from the second cardiac cycle.

Simulations were performed by assuming the blood in the AV apparatus to be a Newtonian fluid, given the size of the vessel. The flow, assumed to be laminar and incompressible, was modeled by Navier-Stokes equations. Blood flow parameters were taken as $\rho=1060 \mathrm{~kg} / \mathrm{m}^{3}$ and dynamic viscosity as $\mu=0.004$ [23].

To model the AV apparatus tissue, identical properties were assumed for the different parts (leaflets, sinuses of Valsalva and ascending aorta). The tissue was modeled as a hyperelastic material with properties: Poisson coefficient $\mu=0.3$, density $\rho=1100 \mathrm{~kg} / \mathrm{m} 3$ and a Young modulus of E=2 MPa.

An unstructured mesh was created for both domains using tetrahedral with Lagrange P2 and Lagrange P2-P1 finite elements for solid and fluid, respectively. Mesh independence tests were conducted for the model with and without coronaries, resulting in a mesh of 201328 elements (solid) and 344495 elements (fluid). The selected mesh for the solid is depicted in Figure 2H. A cluster of 32 CPUs with COMSOL Multiphysics v5.2 was used to run the simulations [6]. 


\subsubsection{Boundary conditions and the coronary pressure model}

Pressure boundary conditions were used for the LVOT level (pLV), ascending aorta level (pAO), and coronary ostia (pCo). The left ventricle and ascending aorta pressure curves were obtained following the CircAdapt model [1] which uses patient-specific parameters (heart rate, systolic blood pressure and diastolic blood pressure). Coronary pressures were characterized from human data extracted from patients who underwent a clinically indicated instantaneous wave-free ratio study (iFR), as shown in the screenshot in Figure 3. To characterize the reconstruction of this boundary condition, we used temporal references based on the ECG signal in the iFR study. Several values were measured at specific key moments - $t_{P}$ (considered as the starting point), $t_{R}$ (when the mitral valve closes), $t_{T}$, and AV closure - at the maximum of the $\mathrm{P}, \mathrm{R}$ and $\mathrm{T}$ waves, respectively. Some extra intermediate values were considered to preserve the shape of the curve (see Table 1). A cubic spline was computed to obtain the complete coronary pressure from the iFR values (see Table 1 and Figure 3). Of the several ways to merge these signals in a common time reference, we used the abovementioned $t_{R}$, which can be identified in the signal when ventricular pressure rises rapidly due to isovolumic contraction effects.

Regarding the boundary conditions for the solid domain, the AV apparatus was assumed to be clamped at the LVOT and aorta endings. The load from the fluid over the cardiac cycle was applied to the interior faces of the geometry where blood and tissue interact as an FSI condition as shown in [25].
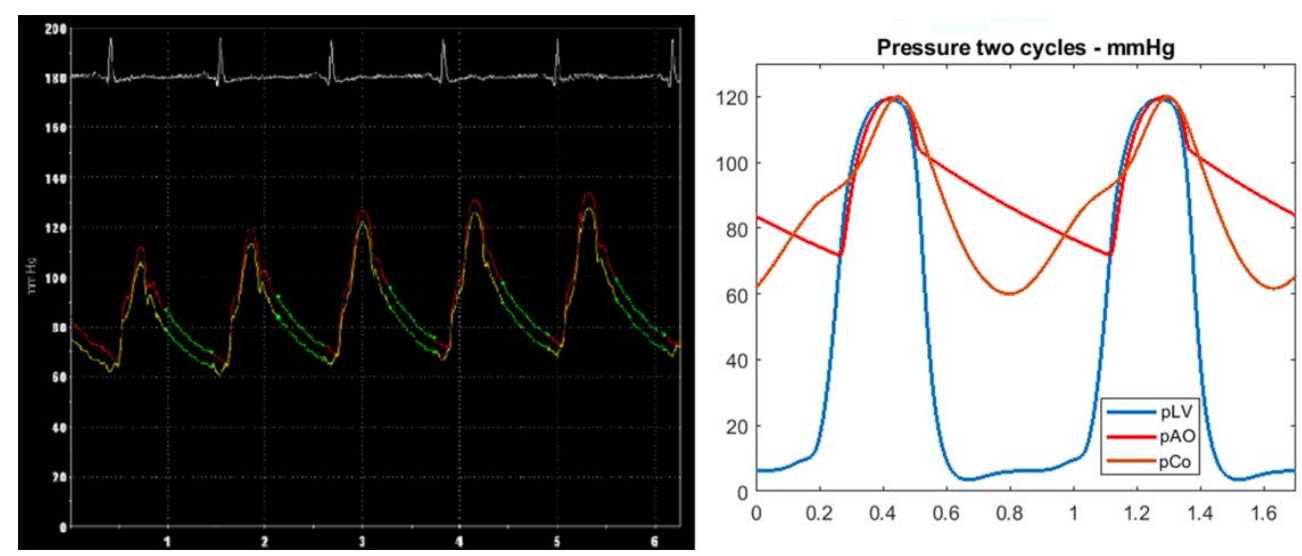

Figure 3: Left: Screenshot of coronary pressure obtained from a patient's iFR study (conducted at Hospital Alvaro Cunqueiro (Vigo) using Verrata pressure guide wire (Volcano - Philips)) and showing the ECG signal (above) and coronary pressure wire signals (red-yellow lines). Right: Boundary condition plot of the pressures used for the numerical simulation of two cardiac cycles. 


\section{RESULTS}

Experiments were conducted to evaluate the novel aspects of the model and the simulation results allowed a comparison from the geometric point of view between the non-coronary model (NCM) and the proposed coronary model (CM). The NCM geometry, unlike the CM geometry, did not consider the coronary arteries and did not include ostium holes. Threeleaflet asymmetry was analyzed via key parameters (displacement, position, and stresses) for the solid domain.

AV opening and closing dynamics during systole were analyzed to compare model kinematics. The geometric orifice area (GOA) — as described elsewhere by Labrosse [21] — and the clinically named AV area [11] were computed by projecting the AV free margin on the annular plane. The area of this projection was obtained by splitting the projection into four triangles and adding the area of those triangles using a script developed in the laboratory. This code enabled analysis of the GOA as a function of time, GOA(t), during the simulated beating cycle. As can be observed in Figure 4, the AV took around $75 \mathrm{~ms}$ to open to a $600 \mathrm{~mm}^{2}$ orifice area in both models, but slope was slightly faster in the NCM. The ejection time (i.e. the time the valve remained open) was around 285 ms for both models, as shown in Table 2, along with valve opening and closing times. Note that we considered the valve to be open when more than half open $\left(G O A>300 \mathrm{~mm}^{2}\right)$. The results for the NCM corroborate previously reported GOA calculations published in the literature [32]. Leaflet behavior for the CM was slightly different to that of the NCM. Two differentiated states of approximately $70 \mathrm{~ms}$ could be distinguished for systolic ejection, i.e. when the area remained at high values, at around $550 \mathrm{~mm}^{2}$ and at around $450 \mathrm{~mm}^{2}$.

Table 2. Comparison of the duration of different systolic ejection phases between the non- and coronary models, various published durations, and in-vivo echocardiography measurements of leaflet dynamics. RVOT: rapid valve opening time; RVCT: rapid valve closing time; ET: ejection time.

\begin{tabular}{|c|c|c|c|c|}
\hline & RVOT (ms) & RVCT (ms) & ET (ms) & $\begin{array}{l}\text { Systolic } \\
\text { phase (ms) }\end{array}$ \\
\hline \multicolumn{5}{|c|}{ Geometrical models that exclude coronary ostia } \\
\hline Non-coronary model & 60 & 45 & 295 & 400 \\
\hline Sturla 2013 - Structural & 56 & 44 & 257 & 357 \\
\hline Sturla 2013 - FSI & 50 & 40 & 260 & 350 \\
\hline \multicolumn{5}{|c|}{ Geometrical models that include coronary ostia } \\
\hline Coronary model & 68 & 57 & 275 & 400 \\
\hline Mohammadi et al. 2016 & 67.1 & 42.8 & 320.2 & 430 \\
\hline Echocardiography [23] & $57.5 \pm 11.1$ & $39.5 \pm 5$ & $329 \pm 63$ & $346.9-505.1$ \\
\hline
\end{tabular}




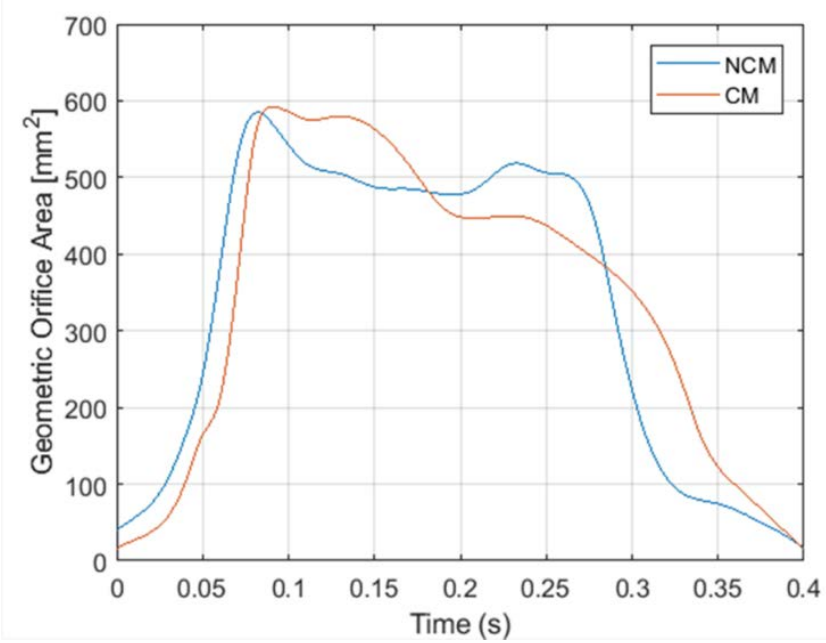

Figure 4: Results for the geometric orifice area (GOA) as a function of time for the coronary model (red) and the non-coronary model (blue) during 0.4 seconds of the simulated heartbeat. Note that since only the systolic phase is of interest, only time ranges from 0 to $0.40 \mathrm{~s}$ are shown. Time 0 corresponds to the moment when the leaflets start to open.
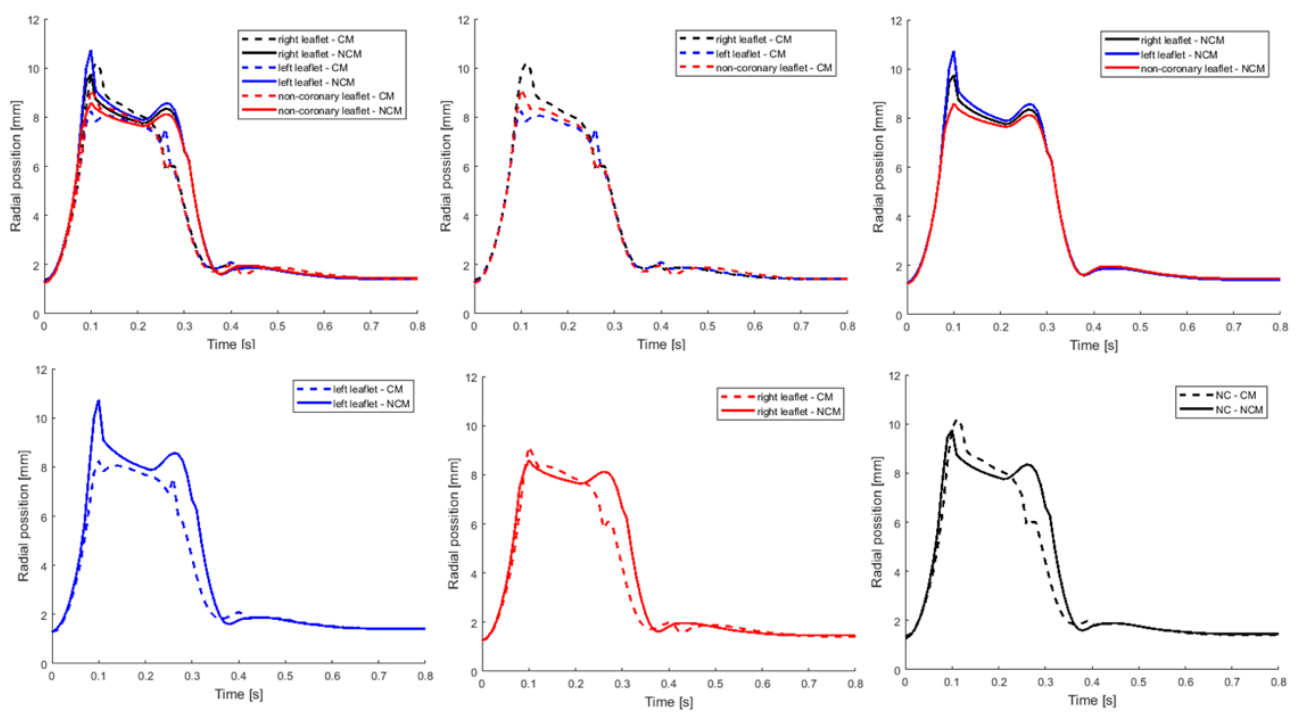

Figure 5: Time-dependent radial positions of the nodulus of Arantius for the coronary model (CM, solid lines) and the non-coronary model (NCM, broken lines). Above: the three leaflets named quantity for the two models. Below, left to right: left leaflet, right leaflet and non-coronary leaflet for both models. 
The maximum displacement of the NCM was $12.3 \mathrm{~mm}$, which corresponds to the moment of maximum valve opening; the corresponding value for the CM was $12.5 \mathrm{~mm}$. The timedependent absolute radial position of the nodule of Arantius (central free margin of leaflets, with increased thickness and nodule) was computed for three leaflets — right, left coronary and non-coronary — as shown in Figure 5 (similar to results published in [40]). The maximum radial position of the nodule of Arantius corresponded to the maximum displacement for both models when the valve was fully opened. Table 3 shows those values. It also shows that there was no displacement variation greater than $2 \%$, as previously observed elsewhere [32].

Table 3. Values and times t for maximum radial position of the nodule of Arantius. NC: non-coronary.

\begin{tabular}{cllllll}
\hline & \multicolumn{2}{l}{ Non-coronary model leaflets } & \multicolumn{3}{l}{ Coronary model leaflets } \\
\cline { 2 - 7 } & Left & Right & NC & Left & Right & NC \\
\hline $\begin{array}{c}\text { Maximum radial } \\
\text { position (mm) } \\
\mathbf{t}(\mathbf{s})\end{array}$ & 11.276 & 12 & 12.4 & 11.5 & 11.986 & 12.51 \\
\hline
\end{tabular}

Maximum principal stress, computed at two different times during opening of the cardiac cycle, as shown in Figure 6, corroborates published results [40]. Some elements near the commissures achieved a maximum principal stress of 2.05 MPa. Since those elements made visualization of stresses in other areas difficult, this problem was solved using a filter to remove those elements (as previously done in [21]). The surface integral of the stress for the entire belly leaflet of each geometry (CM and NCM) was calculated; the maximum obtained values of $4.65 \mathrm{~N}$ and $3 \mathrm{~N}$, respectively, indicate the significant effect of considering our model for this kind of analysis. This phenomenon can also be observed in Figure 7, which depicts the total stress supported by the leaflets and, again, shows a greater difference in the left leaflet for the NCM versus the CM. The CM also shows the blood flow through the coronary ostia, a fact which was not shown in previous models. 


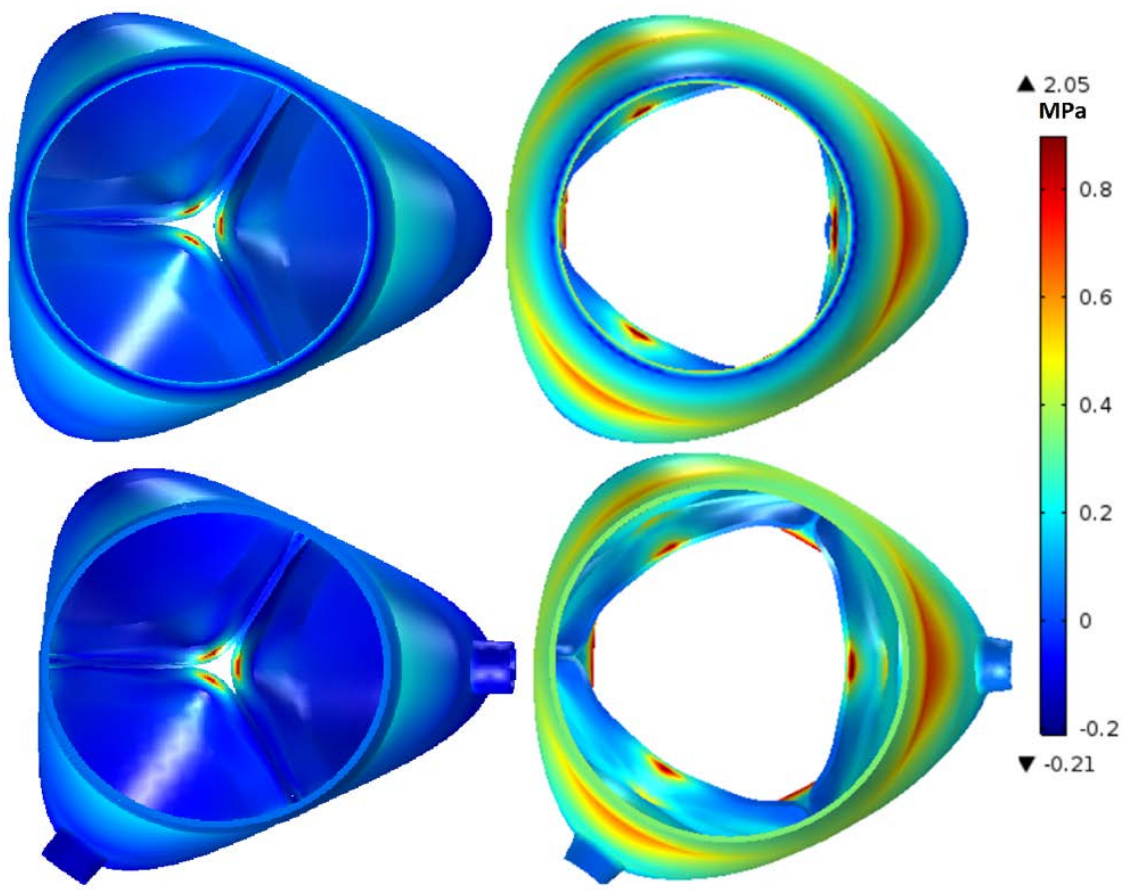

Figure 6: Maximum principal stress distribution throughout the systole at $0.18 \mathrm{~s}$ and $0.31 \mathrm{~s}$ (reading left to right) for the non-coronary model (above) and the coronary model (below).

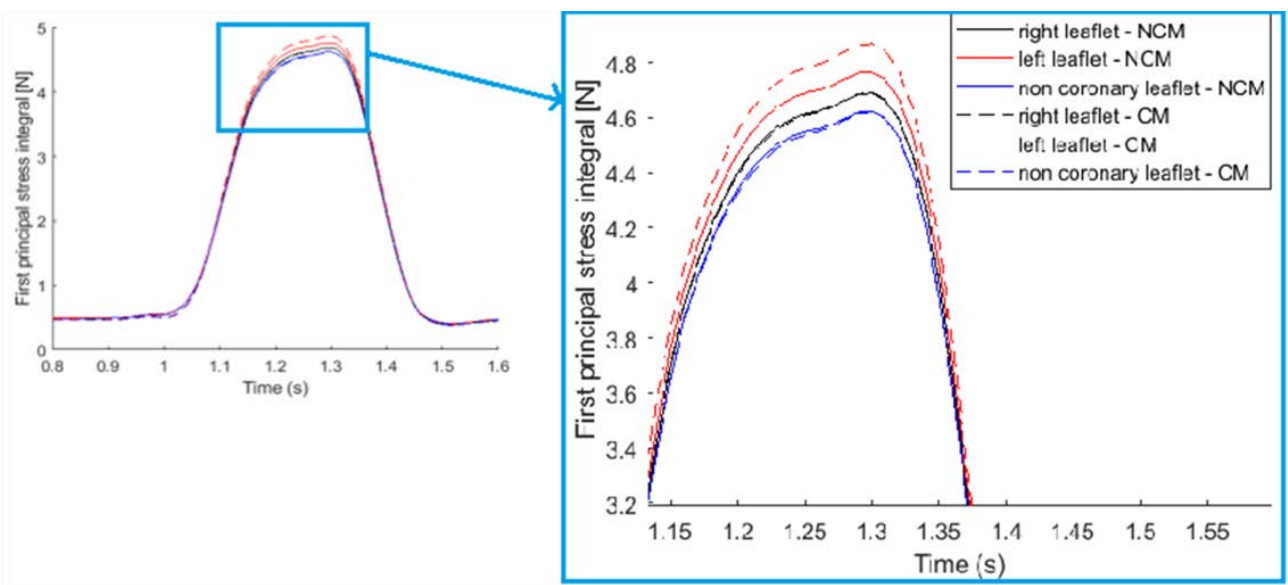

Figure 7: Time analysis of all the stress supported by leaflets for the coronary model (CM) and the noncoronary model (NCM). Left: surface integral of the stress. Right: zoom of the left graph. 


\section{DISCUSSION AND VALIDATION}

Deriving models from echocardiographic images is very important, because, even if more realistic patient-specific reconstructions can be obtained using CT or MRI (as done for example in $[32,45]$ ), those image modalities, unlike echocardiography, are only used in clinical practice for a smaller number of procedures. Although 2D TTE is routinely used for imaging the $\mathrm{AV}$, we used $3 \mathrm{D}+\mathrm{t} \mathrm{TEE}$ echo because it can provide extra and more precise information in the form of a full assessment of the 3D apparatus and characterization of the coronary artery ostia and LVOT. Our new methodology for building a geometric and biomechanical model can reflect the complexity of the entire AV apparatus using routine echocardiographic images.

The geometric construction process required several parameters, some previously known and others resulting from the inclusion of new structures. The thickness parameters thL and thV were included because there is no consensus on a unified and standard wall modeling approach. Some authors consider the leaflets as shell-type elements [7] or resistive Eulerian surfaces [19], whereas other authors treat them as solids [20]. A thickness value for the aortic sinus, between $0.5 \mathrm{~mm}$ [21] and $3 \mathrm{~mm}$ [11], is applied to account for the overall constraining effect of the soft tissues surrounding the AV in vivo. There is no clear rule regarding thickness parameters, as documented in a recent study discussing and comparing leaflet thicknesses [16]. In our geometric model, those parameters - which can be derived from TEE as explained elsewhere [35] - were as close to reality as possible, namely, $0.5 \mathrm{~mm}$ for the leaflets and $1 \mathrm{~mm}$ for the aortic tissue. Further studies are required, nonetheless, in order to determine an optimal value for those parameters.

Assumed for the LVOT was that it is a prolongation of the base of the interleaflet triangles in the 'vertical' direction and that it has a uniform angle $\psi$ along the circumferential plane, as shown in Eq. 1. A further improvement to the model — which, however, has the drawback of also adding complexity - would be to measure this angle in several radial directions to reconstruct the LVOT with different slopes. The required parameters for LVOT construction are $\mathrm{H}_{\mathrm{LVOT}}$ for height and $\mathrm{D}_{\mathrm{LVOT}}$ and the VAj diameter (Da), to build the upper and lower circular sections of a truncated cone.

Concerning our results, NCM geometry and CM geometry were simulated and then compared with physiological data. GOA behavior of the AV was similar for NCM and CM, as shown in Figure 4. Plot shape is very similar to plots published elsewhere [21], where an NCM was simulated and the results compared with those for a laboratory experiment. The maximum radial position for our NCM and CM does not diverge by more than $2 \%$, as shown in Table 3.

The models were evaluated with a numerical study reflecting an intermediate position between a structural and an FSI moving mesh analysis. Values in Figure 5c show the value for the radial displacement of the nodule of Arantius lies in the range of previously published results [40], where a first approach to FSI simulation was to compare it with the structural model. For the numerical simulation, a novel approach was to obtain coronary boundary conditions from a patient-specific iFR study. 
Regarding the total stress supported by the leaflets, there was little difference between the NCM and the CM; the maximum difference was observed for the larger left leaflet. The influence of leaflet asymmetry was also evident: as asymmetry increases, so also does the integral of the stress increase, as shown in Figure 6.

\section{CONCLUSIONS AND LIMITATIONS}

We have described a new biomechanical model of the AV apparatus that includes the ostia and LVOT as new features. The patient-specific model is based on 3D TEE image sequences that can be applied to a large number of patients. Our model aims for a similar precision to models derived from more advanced imaging techniques, as proposed recently [45], but is based on more routinely available and less costly acquisition methods. The model is an improvement on existing previous echo-derived models [20,33,44], incorporating several additional parameters extracted from such images: diameter of the left-right coronary artery, diameter of the LVOT, height under the LVOT, length of the coronary arteries, leaflets thickness, aortic sinus thickness, ascending aorta thickness, LVOT thickness, coronary artery thickness. This model improvement allows a more realistic simulation of the AV biomechanics. We also provide a detailed characterization of the pressure boundary conditions used for the numerical simulation. Our results are like those reported in the literature, although precise comparison is not possible, due to differences in the geometric models and, more importantly, to differences in boundary conditions (these are not usually described in such detail).

The main limitation of this study is that the process is not fully automated; even though the proposed patient-specific model is realistic, some manual intermediate steps are required. Another limitation of the study is the uniform tissue material properties. The simulation could be improved: by distinguishing between leaflet tissue and calcified plaques and by a better characterization of the fluid. Further research is required to validate this new geometric model against CT and MRI data and for different patient conditions.

\section{FUNDING}

Marcos Loureiro-Ga was awarded a PhD scholarship from Xunta de Galicia, partially financed by European Union funds FSE Galicia 2014-2020, with ID number IN606A-2017/029.

\section{CONFLICT OF INTEREST}

Marcos Loureiro-Ga, Cesar Veiga, Generosa Fdez-Manin, Francisco Calvo-Iglesias, Victor Alfonso Jimenez, Pablo Pazos and Andres Iñiguez declare that they have no conflict of interest.

\section{ETHICAL APPROVAL}

Patient-specific data were obtained from patients who underwent clinically indicated TEE imaging at Alvaro Cunqueiro Hospital (Vigo, Spain) under a protocol approved by the local Institutional Review Board. 


\section{REFERENCES}

[1] Arts T, Delhas T, Bovendeerd P, et al. Adaptation to mechanical load determines shape and properties of heart and circulation, the CircAdapt model. Am J Physiol Heart Circ Physiol 2005; 288: 1943-1954.

[2] Bailey, J., Curzen, N. and Bressloff, N. W., 2017. The Impact of Imperfect Frame Deployment and Rotational Orientation on Stress within the Prosthetic Leaflets During Transcatheter Aortic Valve Implantation. J. Biomech. 53: 22-28.

[3] Baumgartner H., JudyHung, Javier Bermejo, John B.Chambers, Edvardsen, Seven Goldstein, Patrizio Lancellotti, Melissa Le Fevre, Fletcher Miller, Catherine M.Otto. Recommendations on the Echocardiographic Assessment of Aortic Valve Stenosis: A Focused Update from the European Association of Cardiovascular Imaging and the American Journal of the American Society of Echocardiography Volume 30, Issue 4, April 2017, Pages 372-392.

[4] Capelli, C., Bosi, G.M., Cerri, E. et al. Patient-specific simulations of transcatheter aortic valve stent implantation. Med Biol Eng Comput 2012; 50: 183.

[5] Charitos EI, Sievers HH. Anatomy of the aortic root: implications for valve-sparing surgery. Annals of Cardiothoracic Surgery. Annals of Cardiothoracic Surgery. 2013;2(1):53-56.

[6] COMSOL Multiphysics ${ }^{\circledR}$ v.5.2. www.comsol.com. COMSOL AB, Stockholm, Sweeden.

[7] Conti CA, Votta E, Della Corte A, et al. Dynamic finite element analysis of the aortic root from MRI-derived parameters. Medical engineering \& physics. 2010;32(2):212-221.

[8] De Hart J,Peters GWM, Schereurs PJG, Baaijens FPT. A two-dimensional fluid-structure interaction model of the aortic valve. Journal of Biomechanics. 2000; 33: 1079-1088.

[9] FreeCAD v. 0.16, http://www freecadweb.org/ Accessed December 4, 2017

[10] Ghista, D.N. Toward an optimum prosthetic trileaflet aortic-valve design. Med. \& biol. Engng. 1976;14:122

[11] Gorlin R, GORLIN SG, Hydraulic formula for calculation of the area of the stenotic mitral valve, other cardiac valves, and central circulatory shunts. Am Heart J. 1951;41(1):1.

[12] Halevi, R., Hamdan, A., Marom, G. et al. Fluid-structure interaction modeling of calcific aortic valve disease using patient-specific three-dimensional calcification scans. Med Biol Eng Comput 2016;54:1683

[13] Haj-Ali R, Marom G, Zekry SB, Rosenfeld M, Raanani E. A general three-dimensional parametric geometry of the native aortic valve and root for biomechanical modeling. Journal of biomechanics. 2012;45(14):2392-2397.

[14] Hsu MC, Kamensky D, Xu F, et al. Dynamic and flud-structure interaction simulations of bioprothetic heart valves using parametric design with T-splines and Fung-type material models. Comput Mech. 2015; 55: 1211-1225.

[15] Ionasec RI, Voigt I, Georgescu B, et al. Patient-Specific Modeling and Quantification of the Aortic and Mitral Valves From 4-D Cardiac CT and TEE. IEEE Transactions on Medical Imaging. 2010;29(9):1636-1651. 
[16] Joda A, Jin Z, Haverich A, Summers J, Korossis S. Multiphysics simulation of the effect of leaflet thickness inhomogeneity and material anisotropy on the stress-strain distribution on the aortic valve. J Biomech. 2016;49(12):2502-2512.

[17] Jones E, Oliphant E, Peterson P, et al. SciPy: Open Source Scientific Tools for Python 2001. http://www.scipy.org/ Accessed December 4, 2017

[18] Kilner PJ, Yang GZ, Mohiaddin RH, Firmin DN, Longmore DB. Helical and retrograde secondary flow patterns in the aortic arch studied by three-directional magnetic resonance velocity mapping. Circulation. 1993;88:2235-2247

[19] Laadhari A, Quarteroni A. Numerical modeling of heart valves using resistive Eulerian surfaces. Int J Numer Meth Biomed Engng. 2016;e02743

[20] Labrosse MR, Beller CJ, Robicsek F, Thubrikar MJ. Geometric modeling of functional trileaflet aortic valves: development and clinical applications. Journal of biomechanics. 2006;39(14):2665-2672.

[21] Labrosse MR, Lobo K, Beller CJ. Structural analysis of the natural AV in dynamics: from unpressurized to physiologically loaded. Journal of biomechanics. 2010;43(10),19161922.

[22] Labrosse MR, Beller CJ, Boodhwani M, Hudson C, Sohmer B. Subject-specific finiteelement modeling of normal aortic valve biomechanics from 3D+t TEE images. Medical image analysis. 2015;20(1):162-172.

[23] Lau KD, Diaz V, Scarmbler P, Burriesci G. Mitral valve dynamics in structural and fluidstructure interaction models. Med Eng Phys 2010; 32: 1057-1064.

[24] Leyh RG, Schmidtke C, Sievers HH, Yacoub MH. Opening and closing characteristics of the aortic valve after different types of valve-preserving surgery. Cirulation. 1999;100(21):2156-2160.

[25] Loureiro-Ga, M., et al. 2017. An Aortic Root Geometric Model, Based on Transesophageal Echocardiographic Image Sequences (TEE), for Biomechanical Simulation. In: Mateos, M., Alonso, P. (Eds.) Computational Mathematics, Numerical Analysis and Applications. Springer, Cham, pp.249-254.

[26] Loureiro-Ga M, Veiga C, Fdez-Manin G, Jimenez VA, Calvo-Iglesias F, Iniguez A (2020). A biomechanical model of the pathological aortic valve: simulation of aortic stenosis. Computer Methods in Biomechanics and Biomedical Engineering, Vol.23:8.

[27] Loureiro-Ga, M., Veiga, C., Fdez-Manin, G., Jimenez, V.A., Juan Salvadores, P., Baz, J.A., Iniguez, A. 2018. Hemodynamics of the left coronary artery after TAVI procedure: a numerical simulation analysis. In Proceedings of the Congress of the European Society of Cardiology.

[28] Lozano R, Naghavi M, Foreman K, et al., Global and regional mortality from 235 causes of death for 20 age groups in 1990 and 2010: a systematic analysis for the Global Burden of Disease Study 2010. The Lancet. 2012;380:2095-2128

[29] Luraghi G., Wu W., De Gaetano F., Rodriguez Matas J.F., Moggridge G.D., Serrani M., Stasiak J., Migliavacca F. Evaluation of an aortic valve prosthesis: Fluid-structure interaction or structural simulation? Journal of Biomechanics. 2017; 58, pp.45-51. 
[30] Luraghi, G., Migliavacca, F., García-González, A. et al. On the Modeling of PatientSpecific Transcatheter Aortic Valve Replacement: A Fluid-Structure Interaction Approach. Cardiovasc Eng Tech (2019) 10: 437.

[31] Marom, G., Kim, HS., Rosenfeld, M. et al. Fully coupled fluid-structure interaction model of congenital bicuspid aortic valves: effect of asymmetry on hemodynamics. Med Biol Eng Comput 2013; 51:839.

[32] Mohammadi H, Cartier R, Mongrain R. 3D physiological model of the AV incorporating small coronary arteries. Int J Numer Meth Biomed Engng. 2017; 33: e2829.

[33] Morganti S, Valentini A, Favalli V, et al. Aortic root 3D parametric morphological model from 2D-echo images. Computers in biology and medicine. 2013;43(12):2196-2204.

[34] Morganti S, Auricchio F, Benson DJ, et al. Patient-specific isogeometric structural analysis of aortic valve closure. Computer Methods in Applied Mechanics and Engineering. 2015; 284: 508-520.

[35] Pearson AC, Guo R, Orsinelli DA, Binkley PF, Pasierski TJ. Transesophageal echocardiographic assessment of the effects of age, gender, and hypertension on thoracic aortic wall size, thickness, and stiffness. American Heart Journal. 1994;128(2):344-351.

[36] Querzoli G, Fortini S, Espa S et al. A laboratory model of the aortic root flow including the coronary arteries. Exp Fluids. 2016; 57: 134.

[37] Robicsek F, Leonardo da Vinci and the Sinuses of Valsalva, Ann Thoruc Surg. 1991;52:328-35

[38] Rocatello, G., De Santis, G., De Bock, S. et al. Optimization of a Transcatheter Heart Valve Frame Using Patient-Specific Computer Simulation. Cardiovasc Eng Tech (2019) 10: 456.

[39] Rotman, O.M., Kovarovic, B., Sadasivan, C. et al. Realistic Vascular Replicator for TAVR Procedures. Cardiovasc Eng Tech (2018) 9: 339.

[40] Sturla F, Votta E, Stevanella M, Conti CA, Redaelli A. Impact of modeling fluid structure interaction in the computational analysis of aortic root biomechanics. Medical Engineering \& Physics. 2013;35(12),1721-1730.

[41] Schneider, C.A., Rasband, W.S., Eliceiri, K.W. "NIH Image to ImageJ: 25 years of image analysis". Nature Methods 9, 671-675, 2012.

[42] Steinman, D.A. \& Migliavacca, F. Editorial: Special Issue on Verification, Validation, and Uncertainty Quantification of Cardiovascular Models: Towards Effective VVUQ for Translating Cardiovascular Modelling to Clinical Utility. Cardiovasc Eng Tech (2018) 9: 539.

[43] Tango, A.M., Salmonsmith, J., Ducci, A. et al. Validation and Extension of a FluidStructure Interaction Model of the Healthy Aortic Valve. Cardiovasc Eng Tech (2018) 9: 739.

[44] Thubrikar MJ. The aortic valve. Boca Raton, FL: CRC Press; 1990.

[45] Votta E, Presicce M, Cella Corte A, et al. A novel approach to the quantification of arotic root in vivo structural mechanics. Int J Numer Meth Biomed Engng. 2017;33:e2849 
[46] Domenichini, F. \& Pedrizzetti, G. Asymptotic Model of Fluid-Tissue Interaction for Mitral Valve Dynamics. Cardiovasc Eng Tech (2015) 6: 95.

[47] Yeh, H.H., Rabkin, S.W. \& Grecov, D. Hemodynamic assessments of the ascending thoracic aortic aneurysm using fluid-structure interaction approach. Med Biol Eng Comput. 2018; 56: 435.

[48] Araz R. Kivi, Nima Sedaghatizadeh, Benjamin S. Cazzolato, Anthony C. Zander, Ross Roberts-Thomson, Adam J. Nelson, Maziar Arjomandi. Fluid structure interaction modelling of aortic valve stenosis: Effects of valve calcification on coronary artery flow and aortic root hemodynamics. Computer Methods and Programs in Biomedicine, 2020. 